\title{
Arbeit und psychische Gesundheit bzw. psychische Erkrankung
}

\author{
Nervenheilkunde 2019; 38(7): 450-473
}

Der Nervenheilkunde ist zu danken, dass sie dem Thema „Arbeit und psychische Erkrankungen " ein Schwerpunktheft widmet. Dies ist ein wichtiges Thema für die Betroffenen, die Psychiatrie und Psychotherapie, die Arbeitswelt und die Gesellschaft. Wir möchten ergänzend noch 2 Aspekte hervorheben, die uns besonders wichtig erscheinen: Nebenwirkungen und Kontextadjustierung.

\section{Nebenwirkungen}

Der Grundtenor vieler fachlicher und vor allem auch politischer Äußerungen ist, dass Arbeit zu Stress führen kann, moderne Arbeit besonders viel Stress macht und dass dies zu einer Zunahme von psychischen Krankheiten führt, weshalb psychischen Belastungen vorgebeugt werden müsse. Aus psychiatrisch-psychotherapeutischer Sicht hat diese wesentlich auf soziologischen $\mathrm{Hy}$ pothesen basierende Annahme und die daraus abgeleitete Ergänzung des $\S 5$ des Arbeitsschutzgesetzes (ArbSchG) durchaus relevante Negativfolgen:

Eine onkologische Krankenschwester kommt zur arbeitsmedizinischen Routineuntersuchung und wird gefragt, ob es belastend sei Patienten sterben zu sehen, ob sie Schichtdienst mache, ob alle Stellen besetzt seien, ob sie Stress habe und abends erschöpft sei (was auch sonst?). Alle Fragen werden mit Ja beantwortet und der völlig gesunden Krankenschwester ein Reha-Aufenthalt empfohlen, was natürlich erzwingt, ihr auch eine psychische Diagnose zu geben. Als sie dort eintrifft erwägt sie bereits ernsthaft, ihren Job aufzugeben, da dieser ja Stress bedeutet. Nach dieser Lesart darf niemand mehr onkologische Patienten pflegen.

Ein Mann mit Herzinfarkt wird im Rahmen der psychokardiologischen Betreuung gefragt, wo er den Herzinfarkt bekommen habe und ob er Stress hatte. Der Patient berichtet, dass er in seinem Büro saß und als Manager ständig Stress habe (was auch sonst?). Als unmittelbare Folge dieser Beratung hat er einen Rentenantrag gestellt, da sein Beruf Stress beinhaltet und kardiologisch gefährlich sei.

Ein depressiver Patient klagt über Insuffizienzerleben am Arbeitsplatz (was auch sonst?). Therapeutenseitig wird diskutiert, ob der Arbeitsplatz Ursache der Depression sein könnte. Der Patient zieht daraus die Schlussfolgerung, seine Lebenszeit-Verbeamtung zu kündigen. Danach ist er weiterhin depressiv, aber kein Beamter mehr.

Im Rahmen eines betrieblichen Gesundheitsmanagements wird ein Seminar zu Stress, der pathogenen Wirkung von Stress und der Wichtigkeit der Stressvermeidung gehalten. Eine Patientin mit hypochondrisch-somatoformer Störung lässt sich daraufhin krankschreiben.

Derartige Beispiele ließen sich noch viele nennen. Die öffentliche Diskussion um die Pathogenität von Arbeit ist in der Gefahr, zu dysfunktionalen Konsequenzen zu führen. Dazu gehört auch, dass Arbeitgeber oder Vorgesetzte von Arbeitsgruppen angeschuldigt und unter Stress gesetzt werden, wenn in ihren Bereichen hohe Arbeitsunfähigkeitszahlen beobachtet werden. Nach diesem Verständnis müssten Behörden, Ministerien, der öffentliche Dienst insgesamt und die dort verantwortlichen Chefinnen die schlimmsten Arbeitgeber der Welt mit den höchsten „Gratifikationsdefiziten“ sein, da dort die höchsten Arbeitsunfähigkeitsraten gefunden werden.

Wie in einem der Beiträge korrekt angemerkt, sprechen aber alle Daten gegen eine Zunahme von psychischen Erkrankungen über die letzten Jahrzehnte hin, was aber zu erwarten wäre, wenn die vorgenannten Kausalitätsannahmen tragen würden. Die Zahl der Arbeitnehmer, die sich am Beruf überlastet fühlen ist zudem deutlich niedriger, als die epidemiologische Grundrate psychisch kranker Menschen in der Vergleichsbevölkerung, was dafür spricht, dass die arbeitenden Menschen gesünder sind als nicht berufstätige, und es gibt durchaus Hinweise, dass Arbeit sogar eine psychisch protektive Wirkung hat. Die von den
Krankenkassen in stetiger Wiederholung vorgelegten Zahlen mit der Behauptung einer Zunahme psychischer Erkrankungen zeigen zunächst einmal nur, dass die einschlägigen Diagnose-Codes häufiger verwendet werden, woraus nicht einmal abgeleitet werden kann, dass sich die Zahl der klinisch erkannten Fälle erhöht hätte [3]. Aus psychiatrischer Sicht ist es zudem eher erstaunlich, dass die Anzahl der ICD-FCodes über die letzten zwei Jahrzehnte hin nicht noch deutlich stärker angestiegen ist. Es gab und gibt immerhin seit Jahren intensive Anti Stigma Bemühungen bzgl. psychischer Erkrankungen. Es wurden ganze Fußballstadien gefüllt, im Radio und der Presse Werbung gemacht und Lehrer, Feuerwehrleute und die Öffentlichkeit geschult. Dennoch ist der Anteil der psychischen Diagnosen an der Gesamtzahl der Arbeitsunfähigkeitsfälle weiterhin deutlich zu gering. Zufriedenstellend wäre, wenn sich diese Zahl um $500 \%$ erhöhen würde, da es immer noch eine erhebliche Rate der Nichterkennung und Nichtbehandlung psychischer Störungen gibt. Die Zunahme der ICD-FNummern in den Krankenkassenstatistiken ist also kein Grund zur Klage, sondern zur Freude. Sie sind schon gar kein Grund, zu behaupten, dass die moderne Arbeitswelt flächendeckend krank mache.

\section{Kontextadjustierung und Person-Environment-Fit}

Wenn von Belastungen am Arbeitsplatz gesprochen wird, dann muss berücksichtigt werden, dass es im Gegensatz zu physikalischen MAK-Werten (maximale Arbeitsplatzkonzentration) keine absolut bestimmbare psychische Belastung gibt, sondern nur eine relative. Als Beispiel sei auf einen weltweit von ca. 80 Millionen Menschen ausgeübten Beruf verwiesen, der verlangt, dass man mit $50 \mathrm{~kg}$ auf dem Rücken auf den Knien durch unwirtliches Gelände robben muss, kilometerweit zu Fuß laufen muss, von Vorgesetzten herumkommandiert wird, in Hitze und Regen übernachten muss, nur aus Dosen ernährt wird, keinen Kontakt zu seiner Familie hat und oben- 
drein in Lebensgefahr schwebt. Das ist der Soldatenberuf. Trainierte Soldaten können das leisten, während viele Behördenmitarbeiter schon nach kurzer Zeit mental und körperlich zusammenbrechen würden. Es geht also um die Relation von Anforderungen einerseits und Fähigkeiten einer Person andererseits, d. h. den „Person-Environment-Fit“ oder nach der ICF [4] um eine „Kontextadjustierung“ $[1,2]$.

Dies hat unmittelbar Bedeutung für den Zusammenhang zwischen psychischer Krankheit und Arbeit. Schizophren Kranke konnten Kartoffel lesen, solange es noch Handarbeit gab. Mit der Maschinisierung der Arbeitswelt wurden sie arbeitsunfähig, da niemand einen schizophren Kranken auf einen Kran lassen würde. Der Kran macht aber nicht krank, sondern erleichtert die Arbeit, ggf. sogar für Rollstuhlfahrer, nicht aber für schizophren Kranke. Seit etwa 10 Jahren gibt es unter Computereinsatz eine verschärfte Qualitätssicherung in der Arbeitswelt. Es macht nicht krank, wenn von Verkäuferinnen im Supermarkt unter Serviceaspekten erwartet und kontrolliert wird, dass sie freundlich zu Kunden sind, an der Wursttheke stehend die Kunden bedienen oder an der Kasse zügig arbeiten. Die Folge ist allerdings, dass Menschen mit hirnorganischen Störungen, Depression, Angststörung, somatoformen Störungen oder Persönlichkeitsstörungen nicht mehr als Verkäuferin arbeiten können, von Psychosekranken ganz zu schweigen.
Nicht der Arbeitsplatz macht krank, sondern psychisch Kranke sind in der modernen serviceorientierten Arbeitswelt nicht mehr zu brauchen, weil dort Soft-Skills verlangt werden, die bei psychischen Störungen eingeschränkt sind [1]. Die hohen $\mathrm{AU}$ - und EU-Raten gehen daher vorrangig zu Lasten affektiver, neurotischer und Persönlichkeitsstörungen.

Die Konsequenz muss also lauten, dass wir nicht von krankmachenden Arbeitsplätzen reden, weil dies falsch und nicht begründet ist und zu erheblichen Negativkonsequenzen auf individueller wie institutioneller Ebene führt. Stattdessen ist das Problem, dass qualitätsgesicherte Arbeitsplätze psychisch Kranke jeder Art ausschließen. Politisch sind „Toleranzarbeitsplätze“ zu fordern (Verkäuferin, die ständig Pause macht und nicht lächelt), was derzeit übrigens fast nur in Behörden zu finden ist und u. a. auch die dortigen erhöhten AU-Raten erklärt.

Autor

Prof. Dr. Michael Linden

Charité Universitätsmedizin Berlin, Medizinische Klinik m. S. Psychosomatik, Leiter der Forschungsgruppe Psychosomatische Rehabilitation Hindenburgdamm 30, 12200 Berlin Tel. 030/84454048; Fax: 030/84454048 michael.linden@charite.de
Literatur

[1] Linden M, Baron S, Muschalla B. et al. Fähigkeitsbeeinträchtigungen bei psychischen Erkrankungen. Diagnostik, Therapie und sozialmedizinische Beurteilung in Anlehnung an das Mini-ICF-APP (222 S.). Bern: Huber; 2014

[2] Linden M. Fähigkeitsbeeinträchtigungen und Teilhabeeinschränkungen. Erfassung und Quantifizierung in der sozialmedizinischen Beurteilung psychischer Störungen. Bundesgesundheitsblatt 2016; 59: 1147-1153

[3] Jacobi F, Linden M. Macht die moderne Arbeitswelt psychisch krank - oder kommen psychisch Kranke in der modernen Arbeitswelt nicht mehr mit? ASU Arbeitsmedizin, Sozialmedizin, Umweltmedizin, 2018; 53 : 530-536

[4] WHO. International Classification of Functioning, Disability and Health: ICF. Geneva: WHO; 2001

\section{Bibliografie}

DOI https://doi.org/10.1055/a-0976-0469 Nervenheilkunde 2020; 39: 86-87 (c) Georg Thieme Verlag KG Stuttgart · New York ISSN 0722-1541 